\title{
Interrelation between mechanical response, strain field, and local free volume evolution in glassy polymers: Seeking the atomistic origin of post-yield softening
}

 \\ ${ }^{1}$ Polymeric Materials Research Group, Department of Materials Science and Engineering, Sharif University of \\ Technology, P.O.B. 11155-9466 Tehran, Iran \\ ${ }^{2}$ Polymer Engineering Department, University of Bayreuth, D-95444 Bayreuth, Germany \\ ${ }^{3}$ Heinz Maier-Leibnitz Zentrum and Physics Department, Technische Universität München, D-85748 Garching, Germany
}

Received 8 May 2017; accepted in revised form 7 August 2017

\begin{abstract}
A set of complementary experiments are used for the first time to elucidate the interrelation between the mechanical properties, the strain field, and the free volume evolution during non-homogenous compression of a glassy polymer. Two sets of quenched and annealed polystyrene samples, having different free volume histories, are notched and exposed to compression. The variation of both the strain field and the free volume are measured on a microscopic scale via digital image correlation in case of strain and Doppler broadening spectroscopy of positron annihilation line in case of free volume measurements. Eventually, the interplay between the local evolution of free-volume, the local strain field, and the mechanical response is investigated throughout the yielding, softening and plateau regions. We found that in all stages of plastic deformation the generated local strain field is positively correlated to the global strain independent of the active mechanism of plastic deformation. Moreover, the local change of free volume is not correlated to the mechanical response of the polymer at the softening stage. Therefore, the free volume evolution should not be responsible for the intrinsic post-yield softening behavior. The easy flow in the plateau region, however, begins at a particular fraction of free volume independent to the thermal history of samples.
\end{abstract}

Keywords: mechanical properties, glassy polymers, plastic deformation, positron annihilation, digital image correlation

\section{Introduction}

Extensive efforts have been devoted to reveal the underlying mechanisms behind the yielding, softening, and hardening phenomena during the course of plastic deformation in polymers [1-5]. These phenomena define the plasticity of a polymer and hence they are of crucial technological importance in industrial applications. The origin of yielding and hardening in polymers are more frequently investigated [1,2, 6]. However, the origin of the post-yield localization, which is known as the intrinsic softening behavior the stress drop after the yield point in the true stress true strain response of the majority of polymers - is less understood [7-10]. Polystyrene (PS) has been widely used as a model material to study the origin of the softening in polymeric systems $[6,11]$. A fullyannealed PS sample, in compression test, experiences yield point followed by a sharp stress drop and then a plateau region in its stress-strain curve. This drastic stress drop is often accompanied by the appearance of micro-shear bands in the microstructure. Quenching a similar PS sample from a temperature above its glass transition point, however, would compromise the post-yield softening response in the 
stress-strain curve and would replace the heterogeneous micro-shear band lines by a diffused-zone of a more homogenous appearance $[12,13]$. While no solid rationale has been given for the difference in plastic deformation behavior of annealed and quenched PS samples, one may rise the importance of free volume on this phenomenon. Note that there is a definite difference in free volume content of annealed and quenched samples.

Several decades ago, the free volume concept became popularized as a means to explain the physical origin, and to quantify, the changes of dynamic and mechanical properties, particularly viscosity, relaxation time, and physical aging in polymeric materials [14-17]. Despite its importance, the correlation between free volume and plastic deformation in solid polymers is unclear and under debate. Early density measurements $[18,19]$ and dilatometry $[20]$ tests revealed that under compression loading, the homogeneous plastic deformation of glassy polymers, particularly PS, is associated with monotonously increase in the density, i.e. the decrease of average free volume content. Later studies by van Melick et al. [21] also supported that the free volume is reduced during the plastic deformation in a homogeneously deformed polymer. When PS samples were rolled to the end of the strain softening region, the overall sample density was increased while the yield stress reduced by almost a factor of two [21]. It is worth to note that the macroscopic densification of polymers during plastic deformation, which is already known [18-21], seems to stand against the idea that the average free volume change in the macroscopic scale controls the plastic deformation mechanism in glassy polymers. However, those free volume evaluations based on macroscopic density measurements could only provide a rough estimation about the average of changes in un-occupied volume which could be different from the local free volume variations in molecular scale. Indeed, atomic experiments based on positron annihilation lifetime spectroscopy (PALS) revealed that an increase in the mean void size and in turn a larger free volume can be achieved due to homogeneous plastic deformation in PMMA samples [22]. According to the findings of the same study, however, after a certain level of homogeneous compression deformation, the increased free volume converged to a constant magnitude in all samples even in those with different initial free volume histories [22]. The hypothesis that an increase in local free volume is responsible for the post-yield softening behavior in polymers received more credit by Molecular Dynamics (MD) simulations conducted by Jatin et al. [2]. They claimed that the post-yield softening is the result of a burst of local free volume evolutions that accompanies a sudden unstable, though local, volume expansion occurring right after the yield point. The local free volume evolution was introduced as a potential mechanism of post-yield softening which could open-up the space in molecular scale for the permanent dihedral flips necessary for conformational changes on the molecular scale - and could facilitate the accommodation of the chains with larger deformations [2]. However, not all atomic experiments support that the free volume expands due to the yielding mechanism in polymers. For instance, Xie et al. [23] performed PALS measurements during uniaxial compression of polycarbonate samples. They found a steady decrease in the average free volume size during homogeneous plastic deformation of polycarbonate.

Most recent numerical studies based on energy landscape analyses suggest that the plasticity of a glassy polymer is controlled by the molecular mobility in the system [24], but there is no solid correlation between the free volume variation and the chain mobility in polymeric systems. In other words, while it is seen that the free volume can be monotonously decreased during the compression test [18-21,25], the molecular mobility can be increased due to the plastic deformation [24, 26, 27]. In conclusion, there is lack of knowledge on how the local free volume actually evolves on a microscopic scale during different stages of the plastic deformation.

In this controversial atmosphere, concurrent experimental evaluation of the local variations of the fraction of free volume and the local strain field can shed light on the topic towards elucidating the atomistic origin that mediates the plastic deformation in polymers. Here, we use digital image correlation (DIC) to visualize the strain field generated on the samples surface during their plastic deformation. A state-ofthe-art positron-based measurement technique, known as Doppler Broadening Spectroscopy (DBS) [28], is used for the first time in order to achieve the contours of local free volume variation in annealed, as well as quenched, polystyrene at different levels of plastic deformation. Eventually, both sets of complementary contour plots are analyzed for better understanding of the relationship between the plastic deformation and the atomic scale evolutions in the glassy polymer. 


\section{Experimental section}

\subsection{Sample preparation and mechanical testing}

The stages of sample preparation are summarized in Figure 1. PS 158 (INEOS Styrolution GmbH) granules were used to produce polystyrene plates through a conventional five step hot pressing process [12] in a stainless steel mold of $100 \times 110 \times 30 \mathrm{~mm}^{3}$ inner dimensions (Figure 1a). The plates were then annealed at $150{ }^{\circ} \mathrm{C}$ to remove the residual stress history, and the homogeneity of the plate was checked by crosspolarized light inspection. The central regions of each plate, showing more uniform molecular orientation, were cut to fabricate samples for a standard compression test. Samples were machined into cuboids of $\sim 19 \times 9 \times 9 \mathrm{~mm}^{3}$ close to the dimensions suggested by Kramer [12] in order to avoid buckling and minimize barreling of the specimens during compression (Figure 1b). Surfaces of each sample were polished with 400, 800, 1200, 2000 and 3000 sand papers, respectively, in order to obtain smooth-enough transparent surfaces proper for free volume measurements. In order to detect the probable free volume evolution within a specific shear band during the plastic deformation, samples were finely notched on one of the lateral surfaces (prism notch with $48^{\circ}$ angle, $1 \mathrm{~mm}$ depth and $\sim 0.1 \mathrm{~mm}$ root radius). The notch was intentionally placed close by the upper surface of each sample instead of the middle, in order to manage the shear band propagation from the notch front at large deformations; while propagation of the upper shear band was geometrically restricted, propagation of the lower shear band was promoted [12]. Since previous free volume studies were mainly conducted under fully homogenous deformation conditions [20, $22,29]$, limited information could be obtained about the correlation between the deformation localization itself and the free volume evolution. The notch, which was introduced to the samples in this study magnifies the localization influence and enables us to provide more insight about the free volume evolution under a more realistic non-homogenous deformation. The samples were then heated above the glass transition temperature $\left(120^{\circ} \mathrm{C}\right)$ for $20 \mathrm{~min}$, and afterwards divided into two separate sets. The $1^{\text {st }}$ set of samples was annealed in the chamber of a DMA instrument (Eplexor, Gabo $\mathrm{GmbH}$ ), which allowed precise controlling of $0.1^{\circ} \mathrm{C} / \mathrm{min}$ cooling rate. The $2^{\text {nd }}$ set of samples was quenched with $10^{\circ} \mathrm{C} / \mathrm{min}$ cooling rate. All samples were stored in a desiccator at room temperature prior to compression test.

A Zwick universal testing machine was used for conducting compression tests under a constant crosshead speed of one $\mathrm{mm} / \mathrm{min}$. The samples were deformed up to three different strain levels, including (I) the yield point, (II) the strain softening point where the work softening mechanism was still dominant - and (III) the plateau region (also called easy flow, glide or cold draw region). A summary of the codings used for each set of samples is provided in Table 1. Four similar samples were tested at each strain level. Strain contour plots were obtained insitu on the deforming surfaces of the samples, while the reported contours represented the local strain distribution right before unloading the samples. No significant variation in the dimensions of the sample

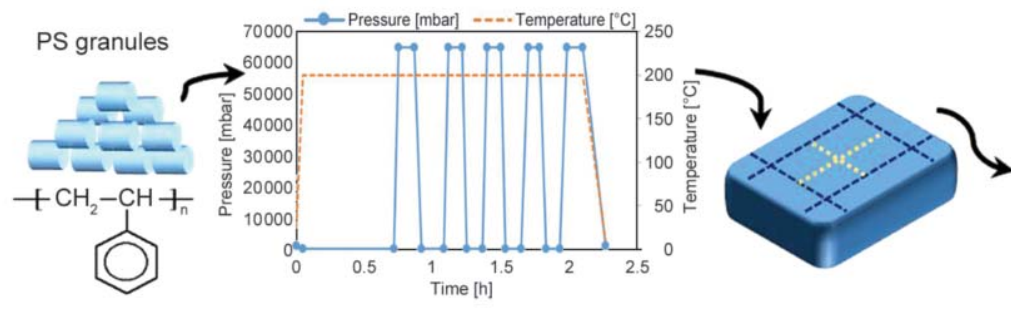

a)

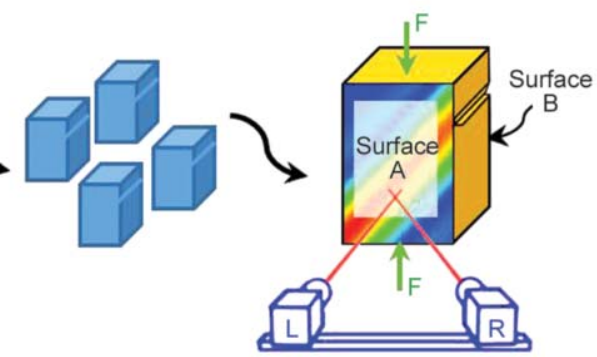

b)

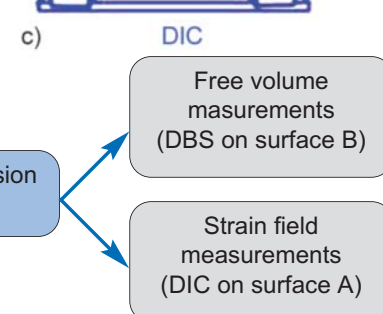

Figure 1. Experimental procedures; a) the 5-stage hot-pressing cycle at constant temperature to produce PS plates, b) the machined and notched samples, c) the setup for DIC measurements 
Table 1. Samples coding and the variables examined in Doppler Broadening Spectroscopy (DBS)

\begin{tabular}{|c|c|c|}
\hline \multirow{2}{*}{ Deformation } & \multicolumn{2}{|c|}{ Heat treatment } \\
\hline & Annealed samples & Quenched samples \\
\hline At the yield point $\left(\varepsilon_{y}\right)$ & $\begin{array}{c}\text { A1 } \\
\text { Time per point: } 30 \mathrm{~s} \\
\text { Step width: } \mathrm{d} x=\mathrm{d} y=0.5 \mathrm{~mm}\end{array}$ & $\begin{array}{c}\text { Q1 } \\
\text { Time per point: } 55 \mathrm{~s} \\
\text { Step width: } \mathrm{d} x=1.5 \mathrm{~mm}, \mathrm{~d} y=0.6 \mathrm{~mm}\end{array}$ \\
\hline At the end of the stress softening regime $\left(\varepsilon_{\mathrm{s}}\right)$ & $\begin{array}{c}\text { A2 } \\
\text { Time per point: } 30 \mathrm{~s} \\
\text { Step width: } \mathrm{d} x=\mathrm{d} y=0.5 \mathrm{~mm}\end{array}$ & $\begin{array}{c}\text { Q2 } \\
\text { Time per point: } 55 \mathrm{~s} \\
\text { Step width: } \mathrm{d} x=1.5 \mathrm{~mm}, \mathrm{~d} y=0.5 \mathrm{~mm}\end{array}$ \\
\hline At the end of the plateau regime $\left(\varepsilon_{\mathrm{p}}\right)$ & $\begin{array}{c}\text { A3 } \\
\text { Time per point: } 55 \mathrm{~s} \\
\text { Step width: } \mathrm{d} x=1.5 \mathrm{~mm}, \mathrm{~d} y=0.6 \mathrm{~mm}\end{array}$ & $\begin{array}{c}\text { Q3 } \\
\text { Time per point: } 55 \mathrm{~s} \\
\text { Step width: } \mathrm{d} x=1.5 \mathrm{~mm}, \mathrm{~d} y=0.6 \mathrm{~mm}\end{array}$ \\
\hline
\end{tabular}

For all measurements, the incident positron beam energy was set $30 \mathrm{keV}$

was observed after finishing the test. At last, one of the four similar samples was isolated, sealed in a waterproof envelope and shipped to FRMII at TUM for performing DBS measurements.

\subsection{Digital image correlation and strain field measurement}

During the compression test, the components of the Green strain tensor on the lateral surface of each sample were obtained by DIC. First, a stochastic black-and-white pattern was sprayed on one of the lateral surfaces of the notched sample (surface A in Figure $1 \mathrm{c}$ of $19 \times 9 \mathrm{~mm}^{2}$ dimensions). Aramis- $2 \mathrm{M}^{\mathrm{TM}}$ analyzer (GOM GmbH, Germany) was then employed to compute the strain contour plots from the digital images recorded by a set of un-parallel cameras (see Figure 1c). The relative movements of subpatterns inside the so-called facet regions, were tracked to calculate the major strain components in the principal coordinate system. The force-displacement data of the universal machine was synchronized to the image acquisition controller. Image acquisition was performed at one-frame-per-second frequency, using conventional $55 \%$ validity quote, $300 \times 300 \mu^{2}$ facet size and $25 \%$ overlap between neighboring facets [30].

\subsection{Doppler-broadening spectroscopy and free volume measurement}

The Doppler-broadening spectrometer located at the NEutron induced POsitron source MUniCh (NEPOMUC) was used for studying the free volume of the regions near the surface of the samples. NEPOMUC holds a universal record in providing one of the highest intensity, slow positron beams of around $10^{9}$ moderated positrons per second with a primary beam energy of $1 \mathrm{keV}[28,31,32]$. The positron beam is guided into the Doppler-broadening spectrometer and focused onto the sample with a beam diameter of around $250 \mu \mathrm{m}[33,34]$. In order to image the lateral distribution of the (nano-)voids in the sample, a region of typically $50-100 \mathrm{~mm}^{2}$ was scanned with $30 \mathrm{keV}$ beam energy. The energy resolution of the Ge detectors was about $1.3 \mathrm{keV}$ and the measurement time for recording a single $\gamma$-ray spectrum was set to 30 or $55 \mathrm{~s}$ (see Table 1). In such a short exposure time, the probable influence of variations of the sample temperature as well as the molecular mobility of the system on the detected signal by the Ge detectors was minimal.

The $511 \mathrm{keV}$ annihilation photo peak was analyzed by means of the commonly used line shape parameters: $S$ and $W$. The $S$-parameter is correlated with those annihilation events with low momentum electrons and in particular with annihilation of parapositronium (p-Ps) which is a hydrogen-like bound state of positron and electron with spins aligned antiparallel. The $S$-parameter is traditionally calculated as the ratio of the integration over the central part of the annihilation line to its total area (see Equation (1)). The $W$-parameter, however, is correlated with those events that arise from direct annihilation of the positrons with core electrons having high momentum and of the so-called 'pick-off' annihilation of ortho-positronium (o-Ps). The $W$ parameter is defined as the ratio of $\gamma$-counts in the wing region of the annihilation peak to the total counts of the annihilation line (see Equation (1)). It is noteworthy that the pick-off process - i.e. the conversion of o-Ps to p-Ps due to the interaction of o-Ps with electrons in the material - and the 'free' positron annihilation (without Ps formation) lead to very similar momentum densities and hence to similar line shapes [28]. Consequently, increased probability for Ps formation and in turn higher p-Ps annihilation rate due to e.g. larger free volume in polystyrene leads to narrower 
annihilation line and hence to higher $S$ (lower $W$ ) parameter.

Note that from a physical standpoint, the free volume is equivalent to the 'total unoccupied space' in the sample. In a simple model the free volume can be described by a distribution of nanometer size spherical voids (see e.g. Tao-Eldrup model $[35,36])$. Therefore, a higher $S$ parameter in this study represents larger free volume or larger fraction of total unoccupied space. Four separate high purity Germanium detectors were incorporated to collect the $\gamma$-ray data for calculating $S$ and $W$ parameters using Equation (1):

$$
\begin{aligned}
& S_{\mathrm{i}}=\frac{\int_{510.1}^{511.9} N(E)_{\mathrm{i}} \mathrm{d} E}{\int_{498.5}^{532.5} N(E)_{\mathrm{i}} \mathrm{d} E}, \quad W_{\mathrm{i}}=\frac{\int_{512.8}^{514.6} N(E)_{\mathrm{i}} \mathrm{d} E}{\int_{498.5}^{532.5} N(E)_{\mathrm{i}} \mathrm{d} E} \\
& S^{\mathrm{xy}}=\frac{\sum_{\mathrm{i}=1}^{4} S_{\mathrm{i}}}{4}, \quad W^{\mathrm{xy}}=\frac{\sum_{\mathrm{i}=1}^{4} W_{\mathrm{i}}}{4}, \quad S / W=\frac{S^{\mathrm{xy}}}{W^{\mathrm{xy}}} \\
& S^{\text {ave }}=\left.\frac{\sum_{\mathrm{k}=1}^{\mathrm{n}_{\Delta \mathrm{y}}} \sum_{\mathrm{j}=1}^{\mathrm{n}_{\Delta \mathrm{x}}} S^{x_{j} y_{k}}}{n_{\Delta \mathrm{x}} \cdot n_{\Delta \mathrm{y}}}\right|_{@ \text { noth-front data points }}
\end{aligned}
$$

where $N(E)_{\mathrm{i}}$ demonstrates the counts of the recorded $\gamma$-rays at energy $E[\mathrm{keV}]$ through the $i^{\text {th }}$ detector, $S_{\mathrm{i}}$ and $W_{\mathrm{i}}$ represent the raw parameters computable from the photopeak of the $i_{\text {th }}$ detector. $S^{\mathrm{xy}}$ and $W^{\mathrm{xy}}$ denote the averaged parameters obtained from all four detectors at each local site on the sample surface which was examined by the spectrometer. Finally, $S^{\text {ave }}$ represents a linear average of $S^{\mathrm{xy}}$ values obtained from those $n_{\Delta \mathrm{x}} \cdot n_{\Delta \mathrm{y}}$ probed points situated inside a window in front of the notch region. Accordingly, one can calculate the variations of the $S$ parameter at the notch-front by excluding the edge effects of the sample or the holder using the shown window in the inset. A summary of other parameters used for obtaining DBS contours is presented in Table 1. The mean penetration depth for monoenergetic positrons having the energy $E$ is given by Equation (2), where $\rho\left[\mathrm{g} \cdot \mathrm{cm}^{-3}\right]$ is the mass density, and $A$ and $n$ are empirical parameters calibrated for the silicon material $\left(A_{\mathrm{Si}}=2.75 \mu \mathrm{g} /\left(\mathrm{cm}^{2} \cdot \mathrm{keV}^{\mathrm{n}}\right), n_{\mathrm{Si}}=1.7\right)[37,38]$. As a rough estimation, assuming similar parameters for polystyrene, we used Equation (3) to approximate the mean positron penetration depth for PS which amounts to $8.6 \mu \mathrm{m}$ for $30 \mathrm{keV}$ positrons used in the current research:

$$
\begin{aligned}
& \bar{Z}=\frac{A}{\rho} E^{\mathrm{n}} \\
& \bar{Z}_{\mathrm{PS}[\mu \mathrm{m}]}=\frac{0.0275}{\rho_{\mathrm{PS}}} E^{1.7}=0.0264 E_{[\mathrm{keV}]} 1.7
\end{aligned}
$$

\section{Results and discussions}

Representative stress-strain curves obtained in compression tests are shown in Figure 2.

A1 and Q1 samples are deformed up to the yield point while $A 2$ and $Q 2$ samples are strained up to the end of the softening regime. A3 and Q3 samples have experienced larger deformation and are strained up to the end of plateau region (usually called easy glide or cold draw region) where the atomistic driving force for the intrinsic softening behavior is saturated. Figures $2 \mathrm{a}$ and $2 \mathrm{~b}$ demonstrate a pronounced

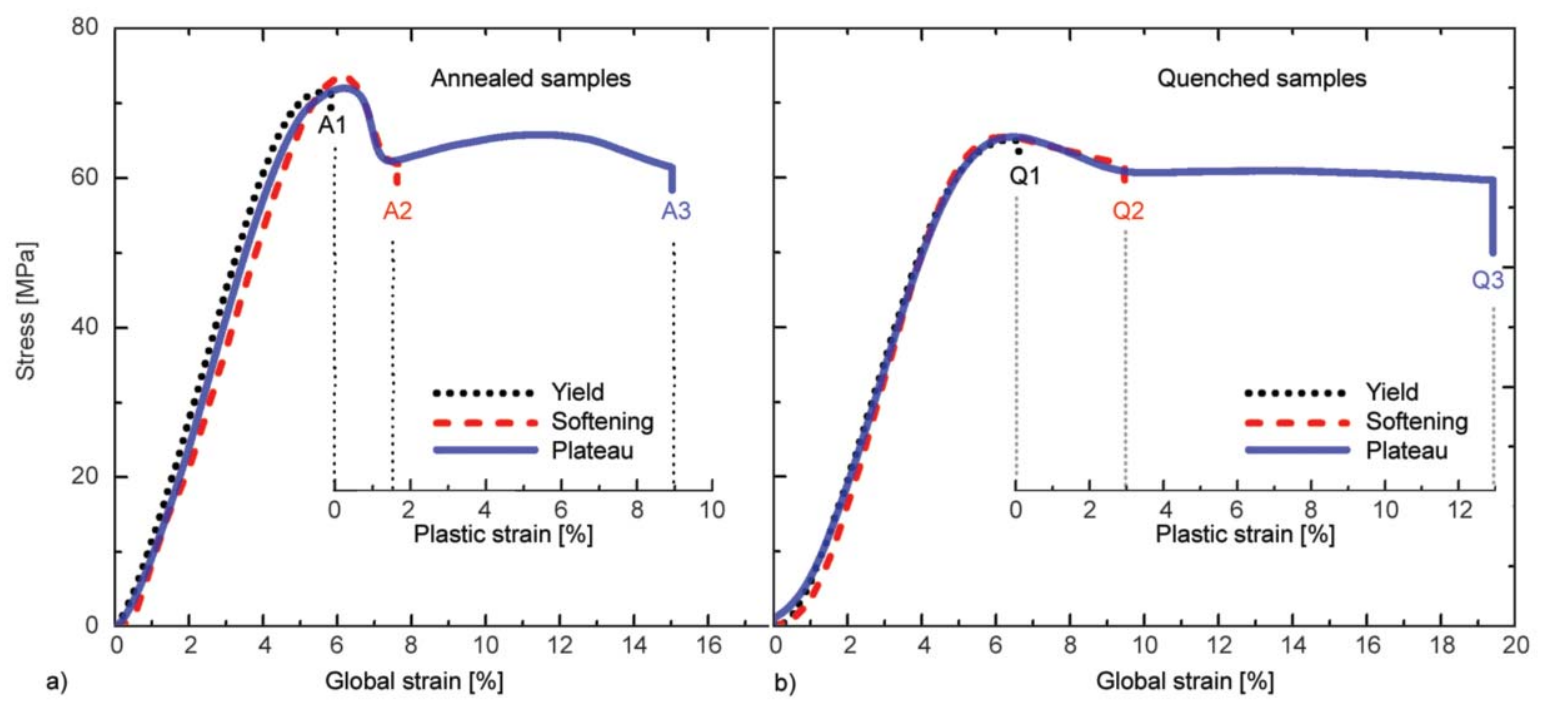

Figure 2. Stress-strain response of notched samples under compression test for (a) annealed and (b) quenched polystyrene samples 
difference between post yielding behaviors of annealed and quenched samples prior to cold draw region. As seen, while the annealed samples show significant stress drop after yield point in the stressstrain curve, the quenched samples show negligible softening behavior. Interestingly, response of the two samples is more or less identical before the yield point (i.e. the Young's modulus) and within the plateau region (i.e. the plateau stress). It is noteworthy that PS naturally shows a small work hardening compared to other polymers like PC and PMMA [6, 7]. In addition, embedding a notch in our samples has encouraged the shear dominant mode - instead of pure compression mode - in large deformations. Consequently, the hardening response is compromised in our samples. Figure 3 compares contour plots obtained by DIC and DBS for the annealed samples exposed to different levels of plastic deformation.

Each DIC contour plot demonstrates the dimensionless major strain and represents the strain distribution of the samples in a macroscopic scale. Each DBS contour plot demonstrates the distribution of ' $S / W$ ' parameter. Although $S$ parameter - which is reported by an arbitrary unit relevant to the used facility - is traditionally used to address the free volume variations, we didn't see a meaningful difference between
$S$ parameter contours and the dimensionless ' $S / W$ ' contour plots. Therefore, in this research we report variations of ' $S / W$ ' parameter to visualize the local variations of the free volume. Figure 4 depicts strain and free volume contour plots for the quenched samples.

Figure 5 summarizes the information in Figure 3a-3c and Figure $4 \mathrm{a}-4 \mathrm{c}$ to elucidate the correlation between the maximum/average of the local strain with the global strain for the annealed, as well as the quenched set. In the annealed set, the strain is drastically localized into a narrow shear band after the yield point. The underlying material experiences $\sim 5$ times larger maximum strain between the yield point and the softening state (where the driving force for softening mechanism is saturated). Further straining of the sample to the end of the plateau region (15\% global strain), results in an increase in the local maximum strain by a factor of $\sim 2.5$ compared to that of the softening saturation state (Figure 5a). In the quenched set, the local strain is gradually distributed in a broader surface area (the so-called diffused zone [12]). The maximum strain between the yield point (6.5\% global strain) and the softening saturation point (9.5\% global strain) is increased by a factor of $\sim 2$. By increasing the deformation to the deformation region where shear mode is dominated $(19.5 \%$ global strain), the maximum local strain is increased

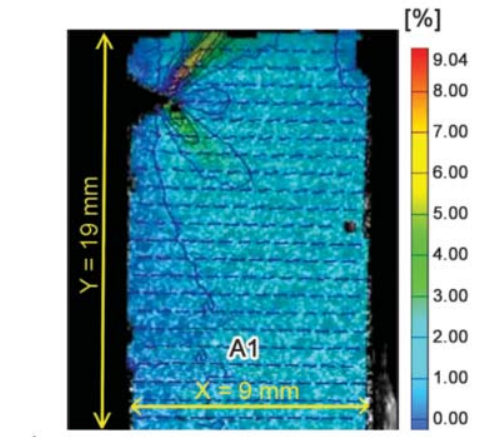

a)

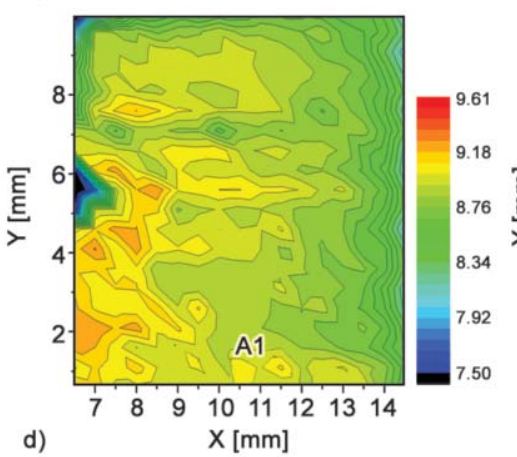

b)
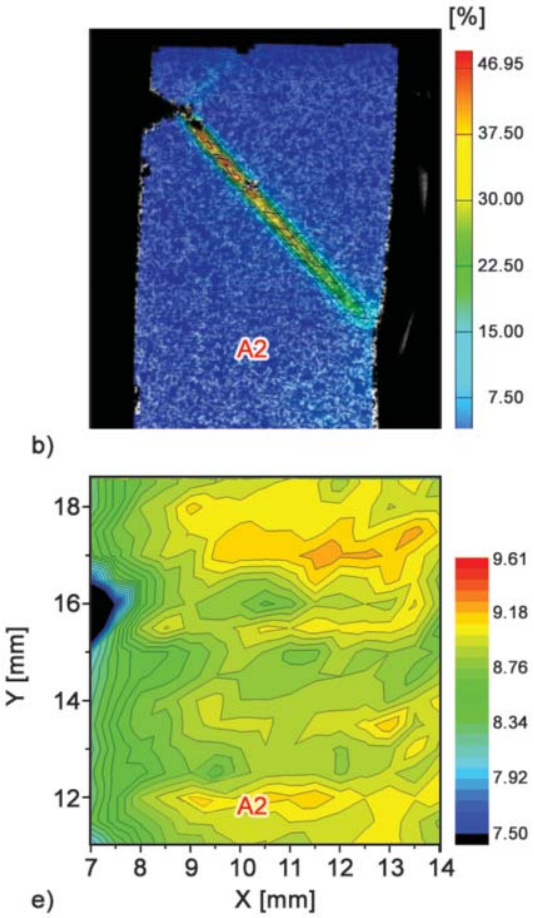

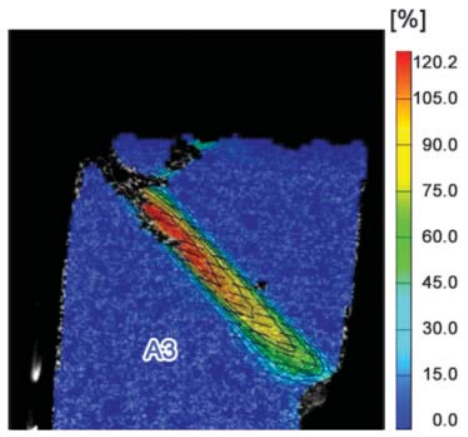

c)

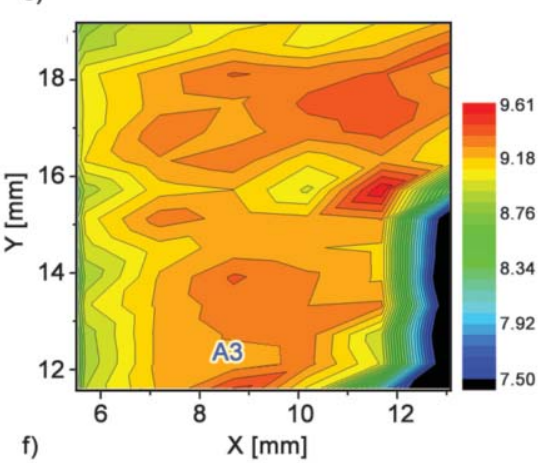

Figure 3. Contour plots of the annealed samples; representing the major strain distribution obtained by DIC for the applied global strains of (a) 6, (b) 7.5, and (c) $15 \%$, respectively; representing the $S / W$ parameter distribution obtained by DBS for the applied deformations of (d) 6 , (e) 7.5 , and (f) $15 \%$, respectively 



b)




c)

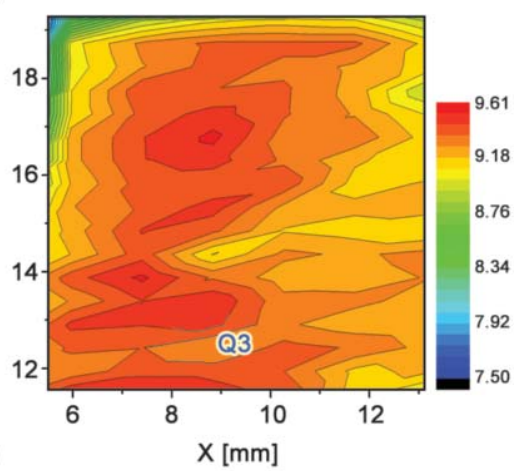

Figure 4. Contour plots of the quenched samples; representing the major strain distribution obtained by DIC for the applied global strains of (a) 6.5 , (b) 9.5 , and (c) $19.5 \%$, respectively; representing the $S / W$ parameter distribution obtained by DBS for the applied deformations of (d) 6.5 , (e) 9.5 , and (f) $19.5 \%$, respectively

again by a factor of $\sim 2$ compared to that of the softening saturation point. Finally, a fully deformed annealed sample locally experiences around 2 times larger maximum strain in comparison with its quenched counterpart. However, the average of local strains in both annealed and quenched samples follow a more or less identical trend with increasing the plastic deformation (Figure 5b). In summary, the generated local strain field in the annealed or quenched sample is positively correlated with the global plastic strain in all stages of the plastic deformation including the yielding, the softening, and the plateau region.

Although DIC could provide components of the strain tensor throughout the deformation region, the contact-less technique established based on a digital

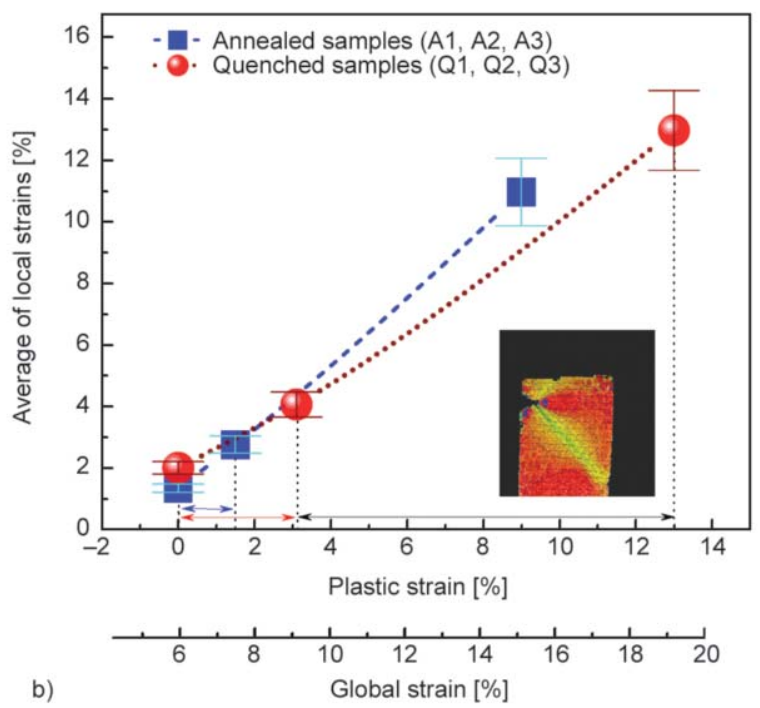

Figure 5. Correlation between variations of (a) the maximum of local major strains and the global strain; (b) the average of local major strains, and the global plastic strain. The local strains obtained from DIC and the global plastic strain (or equivalently the plastic strain) obtained from the universal machine (see Figure 2). 
image correlation algorithm - i.e. DIC [30] - was not able to reveal in-depth information about the atomistic evolutions during the plastic deformation. Therefore, DBS was used as a complementary technique to provide information about variations of the free volume which were shown in Figure $3 d-3 f$ and Figure $4 \mathrm{~d}-4 \mathrm{f}$ for the annealed and the quenched set, repectively. The statistical contour plots obtained by DBS, for the first time, show that the local pattern of free volume evolution is not clearly matched with those of the local strain obtained by DIC. The information about the examined free volume gained by DBS is averaged over the size of the beam spot ( $250 \mu \mathrm{m}$ wide incident positron beam). This might be a potential reason for the differences between the geometry of patterns obtained by DBS and DIC. Interestingly, all samples quenched from above $T_{\mathrm{g}}$ (Figure 4d-4f) show significantly larger free volume in comparison with their annealed counterparts (Figure $3 \mathrm{~d}-3 \mathrm{f}$ ). This observation supports the notion that annealing causes a change in the free volume arrangement by relaxing the meta-stable molecular conformations and decreasing the energy of the system toward a thermodynamic equilibrium. This is while quenching freezes the microstructure of the rubbery state comprising a large fraction of randomly distributed free volume in the system.

According to Figure $3 \mathrm{~d}$, at point $\mathrm{A} 1$ - right after the yield mechanism is activated - the free volume content of the annealed sample is slightly increased close to the notch region. This observation is in accordance with the earlier study which suggested a series of abrupt irreversible dihedral transformations on



segments of the macromolecules in atomistic scale contribute to the yielding mechanism in glassy polymers [2]. Although the evidences of free volume enhancement nearby the notch is observed in the annealed sample at point A1, the relative enhancement of free volume fraction nearby the notch region is not observed in the quenched sample at corresponding point of Q1 (Figure 4d). Since the quenched sample has the maximum practical free volume fraction, therefore initiation of the yielding is not associated with an increment of the free volume in the quenched sample. According to Figures 3 and 4, up to A2 and Q2 points the free volume is not increased anymore, or it maybe redistributing in the sample. However, at points A3 and Q3 (Figures $3 \mathrm{f}$ and 4f), the free volume is again escalated. Indeed, in contrast to the local strain variations, the correlation of the local free volume variation with the applied plastic deformation is not monotonously positive.

Figure 6 reveals more details from DBS measurements. Figure 6a illustrates the variations of the $S$ parameter quantity within a smaller window in front of the notch region (see inset in Figure $6 a$ ) in order to avoid the probable interference of the edge effects. The mean of the $S$ parameter and its measurement error for both annealed and quenched sets are shown with bold lines in this figure. In addition, the domain of variations of the $S$ parameter for all local points examined inside the shown window in front of the notch are hatched with blue and red color for annealed and quenched sets, respectively. Remember that a larger $S$ parameter means a larger free volume fraction in the sample.

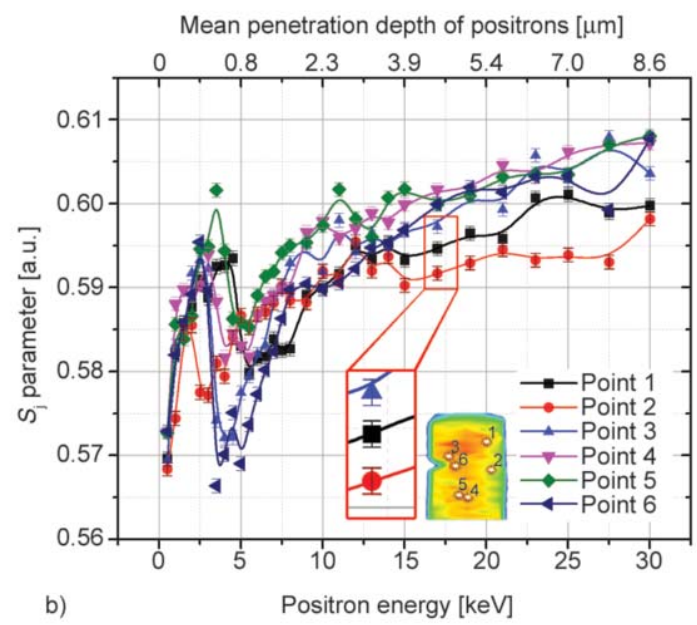

Figure 6. Variation of the $S$ parameter: (a) versus the applied plastic strain for annealed and quenched samples at $E=30 \mathrm{keV}$ beam energy during DBS; hatched areas represent the domain of variations for $S^{\mathrm{xy}}$ parameter obtained from all data points inside the $\Delta x \times \Delta y$ window at the notch-front region; (b) versus the incident positron beam energy $(E)$ 
As seen, in both sets, wherever the softening mechanism is dominant (from $\mathrm{A} 1$ to $\mathrm{A} 2$ in the annealed and from Q1 to Q2 in the quenched samples), the free volume is decreased due to the plastic deformation. The decrease in free volume due to plastic deformation is in accordance with the previous deformation dilatometry and numerical studies [20, 25]. Additionally, note that the free volume drop in quenched samples is significantly higher than the free volume drop in annealed samples. Conversely, the stress drop after yielding in the quenched set was significantly smaller than the stress drop in the annealed set (Figure 2). Since the free volume variation is not correlated with the stress drop variation, this observation shows that the free volume is not responsible for the intrinsic post-yield softening behavior. Interestingly, the thermal history (annealing or quenching treatment) has differently influenced the slope of variations in both mechanical softening (in macroscopic scale) and free volume (in molecular scale). Therefore, the softening should have a thermally activated molecular origin, may be in terms of the molecular mobility - instead of the free volume - of the system.

In both sets, at the plateau region - where the shear mode of deformation is dominant - the free volume is increased due to a probable shear-induced degradation at molecular level (Figure 6a). The shear mode of deformation in this situation could obviously be seen from the local strain contours as well which were obatained by DIC instrument (Figure 3c or Figure 4c). Most importantly, the increase in free volume is proportional to the applied global strain in the shear-dominated region (Figure 6a). Also, it may be noted that free volume is more or less the same at the initiation of easy flow or plateau region (where the stress or load bearing capcity of the material is constant afterwards). Indeed, easy flow starts at a given free volume independent to the thermal history. It seems that after the yield point, the free volume is decreased to adjust to a specific level required for initiation of the easy flow. This is in accordance with another positronbased study that showed during homonenous postyield deformation the total free volume reachs to a constant level independent to the history due to quenching or annealing [22]. Consequently, one should expect a higher slope for free volume reduction in quenched samples during the softening course because the quenched samples have more free volume at the yield point as can be observed in Figure 6a.
In summary, the higher the free volume content (the more open spaces to facilitate permanent dihedral flips necessary for conformational changes [2]), the easier would be the initiation of the yielding. Then, since a given level of stress and thus free volume is required for the initiation of the easy flow, a sharper slope of free volume reduction is expected. This is what is observed for quenched samples. Vice versa, in annealed samples less free volume is present from the beginning. Therefore, higher stress level is required to provide the internal energy for permanent conformational changes and to guarantee activation of the yielding mechanism. Since the free volume is low from the beginning in the annealed set, a lower free volume drop is required for reaching to the level needed for the initiation of easy flow. Thus the commencement of pleatue response is expedited with lower variation in free volume content.

Finally, the precision of DBS measurements is shown in Figure 6b. In this figure, variations of the $S$ parameter is illustrated versus the incident positron beam energy. The acceptable repeatability and high precision of the measurements in different locations on the sample is shown here through the error bars. The mean penetration depth of positrons into the surface is also shown in the upper axis based on Equation (3). The discussed free volume variations in this research are obtained from a depth of around $8.6 \mu \mathrm{m}$ below the sample surface (equivalent to $30 \mathrm{KeV}$ positron beam energy). Figure $6 \mathrm{~b}$ shows that the measured $S$ parameter in this domain has an almost steady magnitude and demonstrates a highly reproducible statistical measurement.

\section{Conclusions}

- The contour plots of the local strain variations, as well as the local free volume variations, on the surface of deformed PS samples were experimentally obtained.

- The local strain variations revealed macroscopic characteristics of the deformation field - i.e. the sharp micro-shear bands in case of annealed samples and the more homogenous diffused-zones in case of quenched specimens - consistent with previous reports in the literature.

- The generated local strain variations in the annealed or the quenched samples were positively correlated with the global strain in all stages of the plastic deformation irrelevant to the mechanism of the deformation. This observation supported that 
the generated local strain filed in the glassy polymer is mostly governed by the geometry of the sample than the active molecular mechanism for the plastic deformation.

- The trend of local free volume evolution, however, was not monotonously correlated with the trend of the local strain nor the globally applied strain. The free volume was slightly increased at the yield point (for the annealed sample), then decreased up to the softening saturation state and finally increased again at the end of plateau region due to the shear-dominated mode of the deformation.

- The free volume drop in the quenched set was significantly higher than the free volume drop in the annealed set. Conversely, the stress drop after yielding in the quenched set was significantly smaller than the stress drop in the annealed set. Since the free volume variation was not correlated to the stress drop variation, the free volume should not be responsible for the atomistic mechanism behind the softening behavior.

- The easy flow or plateau region, however, started at a given free volume independent to the thermal history of samples.

- In quenched samples, presence of a higher initial fraction of free volume expedited the initiation of the yielding at a lower yield stress through providing more space for the permanent conformational changes. Then, it seems that since a given level of free volume was required for the initiation of the easy flow, a drastic reduction of free volume was observed during the post yield softening course in the quenched set. Vice versa was true in case of the annealed samples.

\section{Acknowledgements}

This work is based upon experiments performed at the NEPOMUC facility at FRM II at the Heinz Maier-Leibnitz Zentrum (MLZ), Garching, Germany. The valuable contribution of the reviewers to this manuscript is highly appreciated. Also, the preliminary comments by Amir Fathi and the technical assistance by Jacqueline Uhm at Polymer Engineering Department in University of Bayreuth are strongly appreciated.

\section{References}

[1] van Melick H. G. H., Govaert L. E., Meijer H. E. H.: On the origin of strain hardening in glassy polymers. Polymer, 44, 2493-2502 (2003).

https://doi.org/10.1016/S0032-3861(03)00112-5
[2] Jatin, Sudaroki V., Basu S.: Investigations into the origins of plastic flow and strain hardening in amorphous glassy polymers. International Journal of Plasticity, 56, 139155 (2014).

https://doi.org/10.1016/j.ijplas.2013.11.007

[3] van Breemen L. C. A., Engels T. A. P., Klompen E. T. J., Senden D. J. A., Govaert L. E.: Rate- and temperaturedependent strain softening in solid polymers. Journal of Polymer Science Part B: Polymer Physics, 50, 17571771 (2012).

https://doi.org/10.1002/polb.23199

[4] Quinson R., Perez J., Rink M., Pavan A.: Components of non-elastic deformation in amorphous glassy polymers. Journal of Materials Science, 31, 4387-4394 (1996). https://doi.org/10.1007/BF00356465

[5] Oleinik E. T. F., Rudnev S. N., Salamatina O. B., Kotelyanskii M. I.: Mechanisms of anelastic deformation in solid polymers: Solidlike and liquidlike processes. Polymer Science Series A, 50, 494-506 (2008). https://doi.org/10.1134/S0965545X08050039

[6] Meijer H. E. H., Govaert L. E.: Mechanical performance of polymer systems: The relation between structure and properties. Progress in Polymer Science, 30, 915-938 (2005).

https://doi.org/10.1016/j.progpolymsci.2005.06.009

[7] van Melick H. G. H., Govaert L. E., Meijer H. E. H.: Localisation phenomena in glassy polymers: Influence of thermal and mechanical history. Polymer, 44, 35793591 (2003).

https://doi.org/10.1016/S0032-3861(03)00089-2

[8] Wendlandt M., Tervoort T. A., Suter U. W.: Non-linear, rate-dependent strain-hardening behavior of polymer glasses. Polymer, 46, 11786-11797 (2005).

https://doi.org/10.1016/j.polymer.2005.08.079

[9] Okereke M. I., Buckley C. P., Siviour C. R.: Compression of polypropylene across a wide range of strain rates. Mechanics of Time-Dependent Materials, 16, 361379 (2012).

https://doi.org/10.1007/s11043-012-9167-z

[10] Klompen E. T. J., Engels T. A. P., Govaert L. E., Meijer H. E. H.: Modeling of the postyield response of glassy polymers: Influence of thermomechanical history. Macromolecules, 38, 6997-7008 (2005).

https://doi.org/10.1021/ma050498v

[11] Erichsen J., Nagel C., Günther-Schande K., Rener M., Altstädt V., Faupel F.: Positron annihilation lifetime measurements of mechanically fatigued polystyrene samples. Journal of Polymer Science Part B: Polymer Physics, 46, 1991-1995 (2008). https://doi.org/10.1002/polb.21536

[12] Kramer E. J.: The growth of shear bands in polystyrene. Journal of Polymer Science Part B: Polymer Physics, 13, 509-525 (1975). https://doi.org/10.1002/pol.1975.180130305

[13] Donald A. M., Kramer E. J., Kambour R. P.: Interaction of crazes with pre-existing shear bands in glassy polymers. Journal of Materials Science, 17, 1739-1744 (1982). https://doi.org/10.1007/BF00540802 
[14] White R. P., Lipson J. E. G.: Polymer free volume and its connection to the glass transition. Macromolecules, 49, 3987-4007 (2016).

https://doi.org/10.1021/acs.macromol.6b00215

[15] Robertson R. E., Simha R., Curro J. G.: Free volume and the kinetics of aging of polymer glasses. Macromolecules, 17, 911-919 (1984).

https://doi.org/10.1021/ma00134a064

[16] Voyiadjis G. Z., Samadi-Dooki A.: Constitutive modeling of large inelastic deformation of amorphous polymers: Free volume and shear transformation zone dynamics. Journal of Applied Physics, 119, 2251041/12251041/8 (2016). https://doi.org/10.1063/1.4953355

[17] Li L., Homer E. R., Schuh C. A.: Shear transformation zone dynamics model for metallic glasses incorporating free volume as a state variable. Acta Materialia, 61, 3347-3359 (2013). https://doi.org/10.1016/j.actamat.2013.02.024

[18] Broutman L. J., Krishnakumar S. M.: Cold rolling of polymers 2. Toughness enhancement in amorphous polycarbonates. Polymer Engineering and Science, 14, 249 259 (1974).

https://doi.org/10.1002/pen.760140402

[19] Broutman L. J., Patil R. S.: Cold rolling of polymers. 1 Influence of rolling on properties of amorphous polymer. Polymer Engineering and Science, 11, 165-173 (1971). https://doi.org/10.1002/pen.760110212

[20] van Dijk D. J.: Volume-effects in the yielding of polymeric glasses (in Dutch). PhD Thesis, TU Delft Institutional Repository, Neatherlands (1980).

http://resolver.tudelft.nl/uuid:ef28aee3-4c1c-4ddf-b123$\underline{27 \mathrm{cf} 3 \mathrm{e} 7 \mathrm{a} 4 \mathrm{a} 15}$

[21] van Melick H. G. H., Govaert L. E., Raas B., Nauta W. J., Meijer H. E. H.: Kinetics of ageing and re-embrittlement of mechanically rejuvenated polystyrene. Polymer, 44, 1171-1179 (2003). https://doi.org/10.1016/S0032-3861(02)00863-7

[22] Hasan O. A., Boyce M. C., Li X. S., Berko S.: An investigation of the yield and postyield behavior and corresponding structure of poly(methyl methacrylate). Journal of Polymer Science Part B: Polymer Physics, 31, 185-197 (1993). https://doi.org/10.1002/polb.1993.090310207

[23] Xie L., Gidley D. W., Hristov H. A., Yee A. F.: Evolution of nanometer voids in polycarbonate under mechanical stress and thermal expansion using positron spectroscopy. Journal of Polymer Science Part B: Polymer Physics, 33, 77-84 (1995).

https://doi.org/10.1002/polb.1995.090330109

[24] Chung Y. G., Lacks D. J.: How deformation enhances mobility in a polymer glass. Macromolecules, 45, 44164421 (2012).

https://doi.org/10.1021/ma300431x

[25] Riggleman R. A., Lee H-N., Ediger M. D., de Pablo J. J.: Free volume and finite-size effects in a polymer glass under stress. Physical Review Letters, 99, 1-4 (2007). https://doi.org/10.1103/PhysRevLett.99.215501
[26] Zhou Q-Y., Argon A. S., Cohen R. E.: Enhanced caseII diffusion of diluents into glassy polymers undergoing plastic flow. Polymer, 42, 613-621 (2001). https://doi.org/10.1016/S0032-3861(00)00376-1

[27] Lee H-N., Paeng K., Swallen S. F., Ediger M. D.: Direct measurement of molecular mobility in actively deformed polymer glasses. Science, 323, 231-234 (2009). https://doi.org/10.1126/science. 1165995

[28] Hugenschmidt C.: CDBS: Coincident doppler-broadening spectrometer. Journal of Large-Scale Research Facilities, 1, A23/1-A23/2 (2015).

https://doi.org/10.17815/j1srf-1-50

[29] van Melick H. G. H., van Casteren I. A., Govaert L. E., Meijer H. E. H.: The influence of intrinsic strain softening on the macroscopic deformation behaviour of amorphous polymers. in 'Proceedings of the 15th annual meeting of Polymer Processing Society, 's-Hertogenbosch, The Netherlands' p10 (1999). http://repository.tue.n1/586481

[30] GOM: Aramis user manual software, v6. Braunschweig, Germany (2011).

[31] Hugenschmidt C., Löwe B., Mayer J., Piochacz C., Pikart P., Repper R., Stadlbauer M., Schreckenbach K.: Unprecedented intensity of a low-energy positron beam. Nuclear Instruments and Methods in Physics Research Section A: Accelerators, Spectrometers, Detectors and Associated Equipment, 593, 616-618 (2008). https://doi.org/10.1016/j.nima.2008.05.038

[32] Hugenschmidt C., Piochacz C., Reiner M., Schreckenbach K.: The NEPOMUC upgrade and advanced positron beam experiments. New Journal of Physics, 14, 55027/155027/22 (2012).

https://doi.org/10.1088/1367-2630/14/5/055027

[33] Stadlbauer M., Hugenschmidt C., Schreckenbach K.: New design of the CDB-spectrometer at NEPOMUC for T-dependent defect spectroscopy in $\mathrm{Mg}$. Applied Surface Science, 255, 136-138 (2008). https://doi.org/10.1016/j.apsusc.2008.05.287

[34] Gigl T., Piochacz C., Reiner M., Hugenschmidt C.: Positronbeam for $\mu \mathrm{m}$ resolved coincident doppler broadening spectroscopy at NEPOMUC. Journal of Physics: Conference Series, 505, 12032/1-12032/4 (2014). https://doi.org/10.1088/1742-6596/505/1/012032

[35] Tao S. J.: Positronium annihilation in molecular substances. The Journal of Chemical Physics, 56, 54995510 (1972). https://doi.org/10.1063/1.1677067

[36] Eldrup M., Lightbody D., Sherwood J. N.: The temperature dependence of positron lifetimes in solid pivalic acid. Chemical Physics, 63, 51-58 (1981). https://doi.org/10.1016/0301-0104(81)80307-2

[37] Krause-Rehberg R., Leipner H. S.: Positron annihilation in semiconductors: Defect studies. Springer, Berlin (1999).

[38] Eichler S., Gebauer J., Boerner F., Polity A., KrauseRehberg R.: Defects in silicon after B+ implantation: A study using a positron-beam technique, Rutherford backscattering, secondary neutral mass spectroscopy, and infrared absorption spectroscopy. Physical Review B, 56, 1393-1403 (1997). 